This item was submitted to Loughborough's Research Repository by the author.

Items in Figshare are protected by copyright, with all rights reserved, unless otherwise indicated.

\title{
Psycho-physical limits of interocular blur suppression and its application to asymmetric stereoscopic video delivery
}

\author{
PLEASE CITE THE PUBLISHED VERSION
}

http://dx.doi.org/10.1109/PV.2012.6229734

PUBLISHER

(C) IEEE

VERSION

AM (Accepted Manuscript)

\section{PUBLISHER STATEMENT}

This work is made available according to the conditions of the Creative Commons Attribution-NonCommercialNoDerivatives 4.0 International (CC BY-NC-ND 4.0) licence. Full details of this licence are available at: https://creativecommons.org/licenses/by-nc-nd/4.0/

\section{LICENCE}

CC BY-NC-ND 4.0

\section{REPOSITORY RECORD}

De Silva, Demuni V.S.X., Hemantha Kodikara Arachchi, Erhan Ekmekcioglu, W.A.C. Fernando, Safak Dogan, Ahmet Kondoz, and Saadet S. Savas. 2019. "Psycho-physical Limits of Interocular Blur Suppression and Its Application to Asymmetric Stereoscopic Video Delivery". figshare. https://hdl.handle.net/2134/22774. 


\title{
Psycho-physical Limits of Interocular Blur Suppression and its Application to Asymmetric Stereoscopic Video Delivery
}

\author{
V. De Silva 1 , H. Kodikara Arachchi 1 , E.Ekmekcioglu 1 , A. Fernando 1 , S. Dogan 1 , A. Kondoz and S. Savas $_{2}$ \\ ${ }^{1}$ I-Lab, CVSSP, University of Surrey, Guildford, UK, GU2 7XH, ${ }^{2}$ Koc University, 34450 Sariyer, \\ Istanbul, Turkey. Email:D.Desilva@Surrey.ac.uk
}

\begin{abstract}
It is well known that when the two eyes are provided with two views of different resolutions the overall perception is dominated by the high resolution view. This property, known as binocular suppression, is effectively used to reduce the bit rate required for stereoscopic video delivery, where one view of the stereo pair is encoded at a much lower quality than the other. There have been significant amount of effort in the recent past to measure the just noticeable level of asymmetry between the two views, where asymmetry is achieved by encoding views at two quantization levels. However, encoding artifacts introduce both blurring and blocking artifacts in to the stereo views, which are perceived differently by the human visual system. Therefore, in this paper, we design a set of psycho-physical experiments to measure the just noticeable level of asymmetric blur at various spatial frequencies, luminance contrasts and orientations. The subjective results suggest that humans could tolerate a significant amount of asymmetry introduced by blur, and the level of tolerance is independent of the spatial frequency or luminance contrast. Furthermore, the results of this paper illustrate that when asymmetry is introduced by unequal quantization, the just noticeable level of asymmetry is driven by the blocking artifacts. In general, stereoscopic asymmetry introduced by way of asymmetric blurring is preferred over asymmetric compression. It is expected that the subjective results of this paper will have important use cases in objective measurement of stereoscopic video quality and asymmetric compression and processing of stereoscopic video.
\end{abstract}

Index Terms-Asymmetric coding, Inter ocular blur suppression, stereoscopic video delivery, 3D-TV

\section{INTRODUCTION}

It is unarguable that multimedia systems have improved the quality of life of human beings as a mode of providing entertainment. From the introduction of the radio in the late 19th Century and introduction of analog television in 1926 to the latest home theater systems with 3-Dimensional (3D) television (3DTV) systems at High Definition (HD) resolution, multimedia systems have evolved exponentially during the past century to provide richer content to the users.

One of the major challenges faced in the attempt to deploy advanced 3D Video applications is the high bandwidth required to transmit multiple views simultaneously. One solution to this problem in the case of stereoscopic 3D is asymmetric coding, which makes use of a phenomenon known as "Binocular Suppression”. Accordingly, when one stereoscopic view is encoded at a higher quality and the other view is encoded at a slightly lower quality, the perceived subjective visual quality is dominated by the higher quality view.

There are several forms of Binocular Suppression. If the two eyes are provided with similar images, but of unequal contrast the perception of the Human Visual System (HVS) is dominated by the higher contrast image. This process is known as Interocular Blur Suppression (IBS). Julesz [1] explained the binocular suppression phenomenon with the aid of the experiments he performed with random dot stereograms. According to Julesz, when the low or high frequency (or both) components of the binocular stimuli are identical binocular fusion will arise. On the contrary if the frequency components are different, another form of binocular suppression known as binocular rivalry will occur. In the case of binocular rivalry either one image of the stereo pair is seen or both images are seen alternately.

In Ref. [2], Perkins et al. theoretically analyzed the stereoscopic image compression problem by way of rate-distortion theory. He proposed mixed resolution coding, where the resolution of one image is reduced while the other is kept at its original high resolution. To the best of our knowledge, the first and the only attempt to use the psycho-physical findings of binocular suppression to develop an asymmetric stereoscopic image coder is found in [3]. In Ref. [3] authors use the findings of Liu and Schor [4] regarding the binocular suppression zone to develop a wavelet based encoder that eliminates redundant frequency information from the stereoscopic image pair.

Recently, there have been significant efforts towards identifying the limits for the level of asymmetry or the quality difference with which the stereoscopic images can be compressed [5][6][7]. In Ref. [5] the bounds of asymmetric stereoscopic video compression and its relationship to eye-dominance are examined by way of a user study.

In Ref. [8] by way of subjective experiments authors suggest that when one of the stereoscopic view pair is encoded at a sufficiently high quality (i.e. a PSNR of about $40 \mathrm{~dB}$ ), the other view can be encoded at a lower quality above a display dependent threshold without subjective visual quality degradation. This lower quality threshold or the just noticeable level of asymmetry is around $31 \mathrm{~dB}$ for a parallax barrier display and $33 \mathrm{~dB}$ for a polarized projection display.

In an asymmetric coding scenario, Most of the asymmetric 
coding techniques discussed above are based on subjective experiments performed under different conditions and sequences with different characteristics. Unfortunately, most of the techniques discussed earlier do not explicitly consider the psycho-physical phenomenon underlying asymmetric coding. Besides, the phenomenon of interocular blur suppression has been investigated especially in mono-vision correction related studies [9] that cannot be directly used in asymmetric coding. To address the above issues, a set of psycho-physical experiments are performed to identify the thresholds of interocular blur suppression.

The specific contributions of this paper can be listed as follows,

- A psycho-physical experiment is designed to investigate the maximum tolerance of blur in one view of a stereo pair. The variation of this tolerance with spatial frequency, contrast and orientation is identified.

- Based on the psycho-physical limits, we investigate the theoretical limits of bit rate reductions that is achievable with asymmetric blurring.

- Subjective experiment is performed to compare and contrast blurring and compression as two processing mechanisms to achieve asymmetry in stereoscopic images.

The rest of the paper is organized as follows. The section II presents the psycho-physical experiments that are performed to investigate the limits of IBS. The section III, details a subjective experiment performed to compare asymmetric blurring and asymmetric compression of stereoscopic images. The implications of the subjective results are discussed in section IV and section V concludes the paper with some directions for future work.

\section{ANAlysis Of Tolerance LeVels of Interocular BLUR SUPPRESSION}

This section describes the psycho-physical experiments that are carried out to measure the tolerance levels of IBS. The first experiment investigates the variation of tolerance with varying spatial frequency. The objective of the second experiment is to analyze the variation of IBS tolerance with varying contrast levels. The results obtained from the experiments are also discussed within this section.

\section{A. Experimental Setup}

1) Equipment: The first experiment is performed on a 46" JVC stereoscopic display with passive polarization glasses (Model number GD-463D10). The display resolution is $1920 \times 1080$ and the viewing distance is set at $2.3 \mathrm{~m}$ from the screen. The contrast ratio of this display is 2000:1. This display accepts content based on the left and right representation of 3D video. The effective screen size is $1018 \mathrm{~mm} \times 573 \mathrm{~mm}$, which makes one pixel to be approximately $0.53 \mathrm{~mm} \times 0.53 \mathrm{~mm}$.

2) Subjects: A total of 16 male subjects participate in the subjective experiments. All of them are aged between 21 to 36 years, with a mean of around 28 years. All participants have a good visual acuity $(>0.7$, as tested with a Snellen eye chart), good stereo vision $(<30$ seconds of arc, as tested with

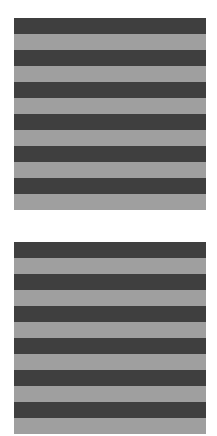

Figure 1. An example of a test stimulus as viewed by the subjects

the Randot stereo test) and good color vision (as tested with the Ishihare test). The sighting dominant eye of each subject is determined by the hole in the card test.

3) Stimuli and Conditions: The main test stimulus is a pair of square wave gratings as shown in figure 1. The stimulus as given in figure 1 is constituted by a stereoscopic image pair. The top square wave gratings are kept unchanged, while one of view of the stereo pair that constitutes the bottom square wave gratings is gradually blurred using a Gaussian low pass filter. The standard deviation of the Gaussian filter $(\sigma)$ is increased in steps of 0.1 per every second. The spatial frequency and the contrast of the stimuli are kept unchanged throughout each reading. The experiments are performed at different spatial frequencies and contrast levels. The subjects will indicate when they perceive a difference in the bottom gratings with relative to the top gratings.

When displayed on the screen, each of the gratings (as shown in figure 1) is a square area with each side measuring $24 \mathrm{~cm}$. When observed at a distance of $2.3 \mathrm{~m}$ from the screen, a set of gratings corresponds to a visual angle of $6^{\circ}$. The spatial frequency of a stimulus is measured by the number of cycles per visual angle $\left(c d e g^{-1}\right)$. In other words, how many times the luminance values alternate within a visual angle of one degree.

The Michelson contrast $\gamma$ as given in Eq. (1) is used to define luminance contrast of stimuli. In Eq. (1), $L_{\max }$ and $L_{\text {min }}$ refer to the maximum and minimum luminance levels present in the stimuli.

$$
\gamma=\frac{L_{\max }-L_{\min }}{L_{\max }+L_{\min }}
$$

All the lighting in the test room is turned off, and the ambient illumination is measured at 5lux.

4) Evaluation of Results: The tolerance level of IBS is presented as the maximum level of blur that could be tolerated. The standard deviation of the Gaussian filter $(\sigma)$ at the maximum tolerable level of blur is used as the measure of IBS tolerance.

To illustrate the relative variation of the tolerance with different frequencies/contrasts the level of IBS tolerance of an individual $\mathrm{j}$ is normalized as given in Eq. (2). 


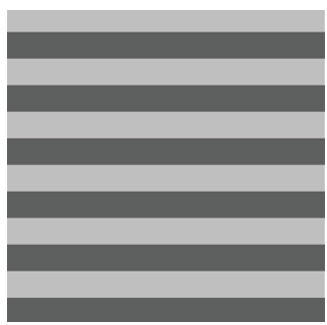

(a) Horizontal $1 \mathrm{c} \mathrm{deg}^{-1}$

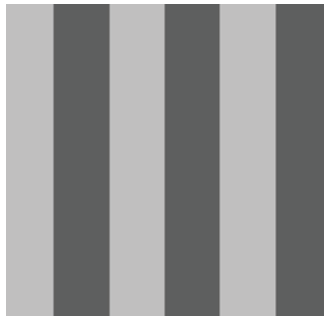

(c) Vertical $0.5 \mathrm{c} \mathrm{deg}^{-1}$

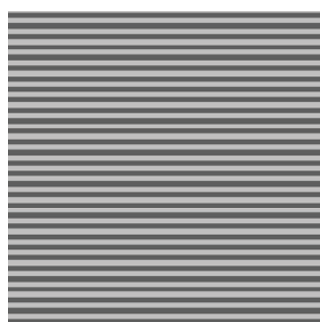

(b) Horizontal $5 \mathrm{c} \mathrm{deg}^{-1}$

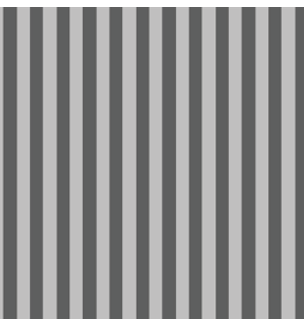

(d) Vertical $2 \mathrm{c} \mathrm{deg}^{-1}$
Figure 2. Examples of test stimuli used to investigate the effect of spatial frequency on IBS tolerance

$$
r_{i, j}=\frac{o_{i, j}-o_{\max , j}}{o_{\max , j}-o_{\min , j}}
$$

In Eq. (2), $o_{i, j}$ refers to the IBS tolerance of individual $j$ to a stimuli $i$ and $o_{\max , j}$ and $o_{\min , j}$ refers to the maximum and minimum values of subject $j$ for all the stimuli in the particular experiment. Thus, $r_{i, j}$ refers to the relative tolerance of an individual $i$ to a particular stimuli $j$.

\section{B. Effect of spatial frequency on IBS tolerance}

This experiment investigates whether the tolerance levels of IBS is affected by the spatial frequency of the content.

The tolerance level is measured at spatial frequencies of 0.3 , $0.5,1,2$ and $5 \mathrm{cdeg}^{-1}$. The tolerance is measured for both horizontal and vertical gratings at each frequency. At a time the bottom gratings of one view of the stereoscopic image pair is gradually blurred and the experiment is repeated by blurring the other view in a similar way. Thus, there are a total of 20 stimuli used in this experiment. The contrast is kept constant for each of the stimuli at 0.3 . The figure 2 illustrates few of the stimuli used in this experiment.

The figure 3 summarizes the average relative tolerance of IBS at different spatial frequencies for vertical and horizontal gratings. In general, subjects can tolerate more blur in horizontal spatial frequencies than vertical frequencies. The psycho-physical tolerance level, in terms of $\sigma$, across different frequencies vary between 3.3 and 2.8 , which is a relatively low variation, considering the width $(3 \sigma)$ of the filter.

\section{Effect of luminance contrast on IBS tolerance}

This experiment investigates whether the tolerance levels of IBS is affected by the luminance contrast of the content.

The tolerance level is measured at luminance contrasts of $0.05,0.11,0.33,0.64$ and 1 . As in previous experiment there

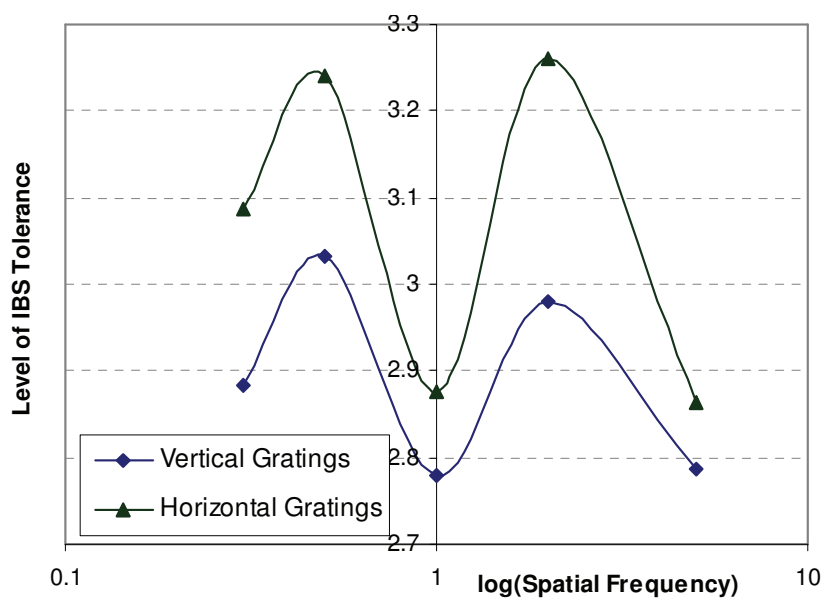

Figure 3. Average tolerance of IBS at different spatial frequencies

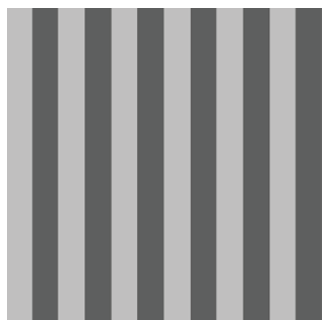

(a) Contrast 0.33

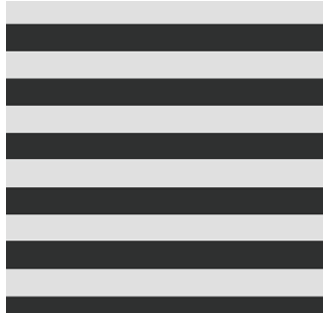

(c) Contrast 0.64

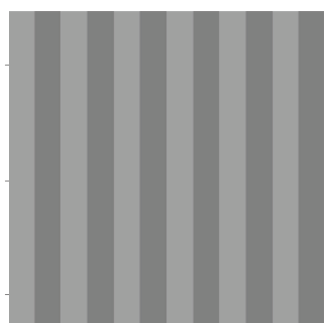

(b) Contrast 0.11

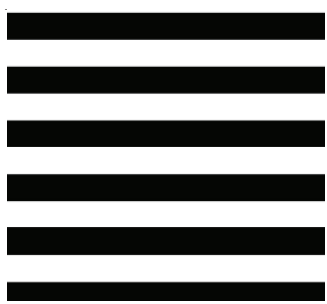

(d) Contrast 1
Figure 4. Examples of test stimuli used to investigate the effect of luminance contrast on IBS tolerance

are a total of 20 stimuli used in this experiment corresponding to the five contrast levels utilized. The spatial frequency is kept constant for each of the stimuli at $1 \mathrm{c} \mathrm{deg}^{-1}$. The figure 4 illustrates few of the stimuli used in this experiment. Similar to the variation of IBS tolerance at different frequencies, the vertical frequencies have low tolerance than horizontal frequencies. However, at very low contrasts $(<0.1)$ tolerance of IBS in vertical frequencies is much higher than horizontal frequencies.

\section{COMPARISON OF INTEROCULAR BLUR SUPPRESSION AND COMPRESSION ARTIFACT SUPPRESSION}

This section describes the a subjective experiment that is carried out to compare and contrast the effect of blurring and quantization processes towards asymmetric stereoscopic image perception. 


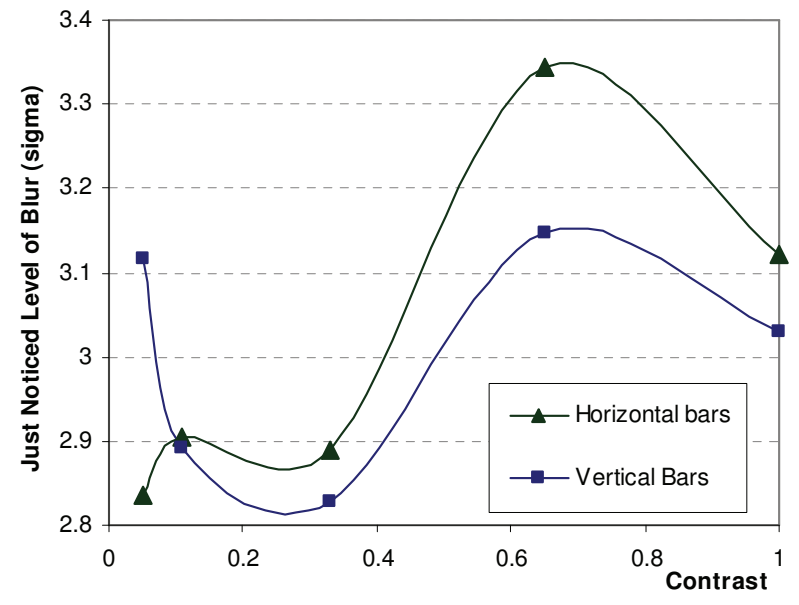

Figure 5. Average tolerance of IBS at different luminance contrasts

\section{A. Experimental Setup}

In this experiment subjects evaluate 8 asymmetric stereoscopic image pairs, 4 of whose asymmetry is achieved by blurring and the other 4 of whose asymmetry is achieved by compression. 16 male subjects, aged 23-40, with normal or corrected visual acuity, participate in this experiment. 4 images of resolution $1920 \times 1080$ are displayed on the same JVC display discussed in section 2.1. The illumination of the viewing environment is 20lux (very dark).

For compression we use the Intra frame encoder of the H.264 Joint Model (JM) reference software version 15.1, the quantization parameter is increased at steps of 1 . For blurring we use a Gaussian low pass filter of 30 pixels wide, and the standard deviation is increased at steps of 0.1 . At the beginning of each sequence, subjects see a symmetric stereoscopic image, i.e. the left and right images are both the uncompressed image. Then the subjects navigate forward of the video sequence, one frame at a time until they notice a difference in quality from the beginning. At each step, either the quantization parameter $(\mathrm{QP})$ or the standard deviation (Stdev) of the Gaussian filter applied on the right view is increased, while the left view is kept unchanged. At the just noticed level of perceived difference, the software records the frame number, where this frame number corresponds either to the QP or to Stdev of the right view at the just noticeable perception difference.

\section{B. Subjective Results}

The results of this subjective experiment are summarized in terms of QP or Stdev of the Gaussian filter at the point of just noticeable perception difference, and the corresponding PSNR and the bit rate of the right view. To provide an indicative bit rate, in the case of Gaussian blurring, the right view at the point of just noticeable difference (JND) is encoded using the JM Intra coder to a PSNR of approximately 40dB. The PSNR of $40 \mathrm{~dB}$ is selected as the benchmark quality achievable by the JM Intra coder that does not yield visually discernible artifacts, which is the same value used in [7] to encode the
Table I

SubJeCTIVE COMPaRISON OF JUST NOTICEABLE LEVEL OF GAUSSIAN BLURRING AND QUANTIZATION

\begin{tabular}{|l|c|c|c|c|c|c|}
\hline \multirow{2}{*}{ Image } & \multicolumn{3}{|c|}{ Quantization } & \multicolumn{3}{c|}{ Gaussian Blurring } \\
\cline { 2 - 7 } & QP & $\begin{array}{c}\text { PSNR } \\
(\mathrm{dB})\end{array}$ & $\begin{array}{c}\text { Bit rate } \\
(\mathrm{kbps})\end{array}$ & Stdv & $\begin{array}{c}\text { PSNR } \\
(\mathrm{dB})\end{array}$ & $\begin{array}{c}\text { Bit rate } \\
(\mathrm{kbps})\end{array}$ \\
\hline Badminton & 43 & 29.44 & 5159 & 5.9 & 16.67 & 3963 \\
\hline Beergarden & 39 & 30.96 & 7017 & 5.4 & 22.52 & 2288 \\
\hline Cafe & 40 & 36.65 & 1692 & 5.6 & 26.27 & 1022 \\
\hline GT Fly & 32 & 38.74 & 4420 & 5.3 & 28.36 & 561 \\
\hline
\end{tabular}

high quality view of the asymmetric pair. The results of this experiment are summarized in Table I.

The results presented in Table I indicate that higher level of asymmetry in terms of PSNR difference or bit rate difference between the stereoscopic pair could be achieved by Gaussian blurring.

\section{Discussion of Subjective Results}

In section II we subjectively evaluated the IBS tolerance at various spatial frequencies and contrasts. Based on the subjective results, in this section we assess the bit rate reductions that are achievable with asymmetric blurring. Furthermore, we analyze the effect of ocular dominance on IBS tolerance level. The section is concluded by contrasting the asymmetric blurring and asymmetric coding of stereoscopic images/videos.

\section{A. Achievable bit rate reductions by asymmetric blurring}

For this analysis we utilize the rate distortion function for source coding a memoryless Gaussian source [10] given as,

$$
R(D)=1 / 2 \cdot \log _{2}\left(\frac{\sigma_{x}^{2}}{D}\right)
$$

In Eq. (3), $\sigma_{x}^{2}$ is the variance of the source and $D$ is the distortion and $R(D)$ is the bit rate at distortion $D$.

The bit rate reductions ( $\Delta R$ in Eq. (4)) achievable by asymmetric blurring of content is calculated based on the Eq. (3). Furthermore, the percentage of average power reduction $(\Delta P)$ is also calculated, as follows,

$$
\begin{gathered}
\Delta R=1 / 2 \cdot \log _{2}\left(\frac{\sigma_{o}^{2}}{\sigma_{b}^{2}}\right) \\
\Delta P=\left(\frac{\sigma_{o}^{2}-\sigma_{b}^{2}}{\sigma_{o}^{2}}\right) \cdot 100
\end{gathered}
$$

where, $\sigma_{o}^{2}$ is the variance of the original source and $\sigma_{b}^{2}$ is the variance of the blurred source.

The $\Delta R$ and $\Delta P$ over various spatial frequencies are presented in Fig. 6, and over luminance contrasts are presented in Fig. 7. As illustrated in Fig. 6, a significant bit rate savings could be achieved by blurring or by resolution reduction for medium to high spatial frequencies. However, according to Fig. 7, at low contrasts, blurring or resolution reduction is not suitable. Furthermore, bit rate savings that could be achieved by blurring is is constant across medium to high luminance contrast levels. 


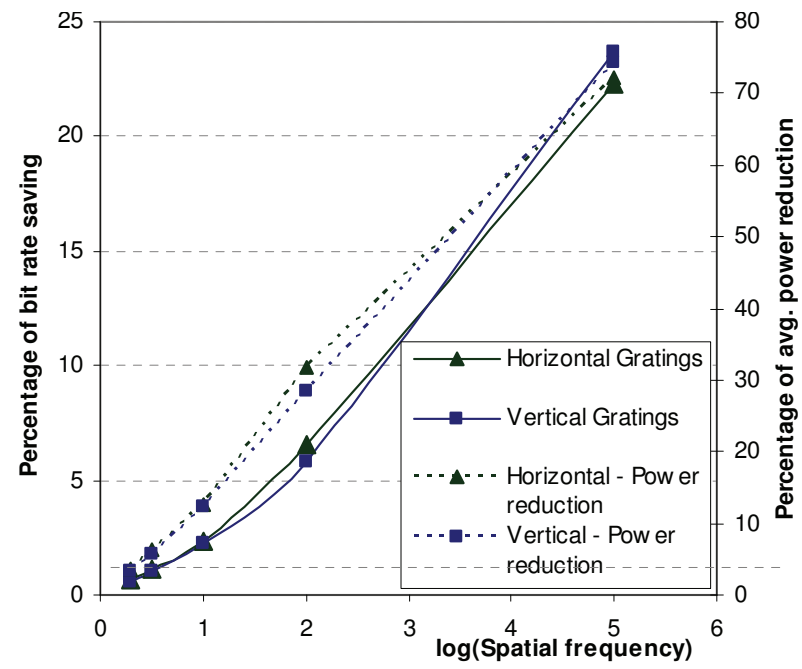

Figure 6. Average tolerance of IBS at different spatial frequencies

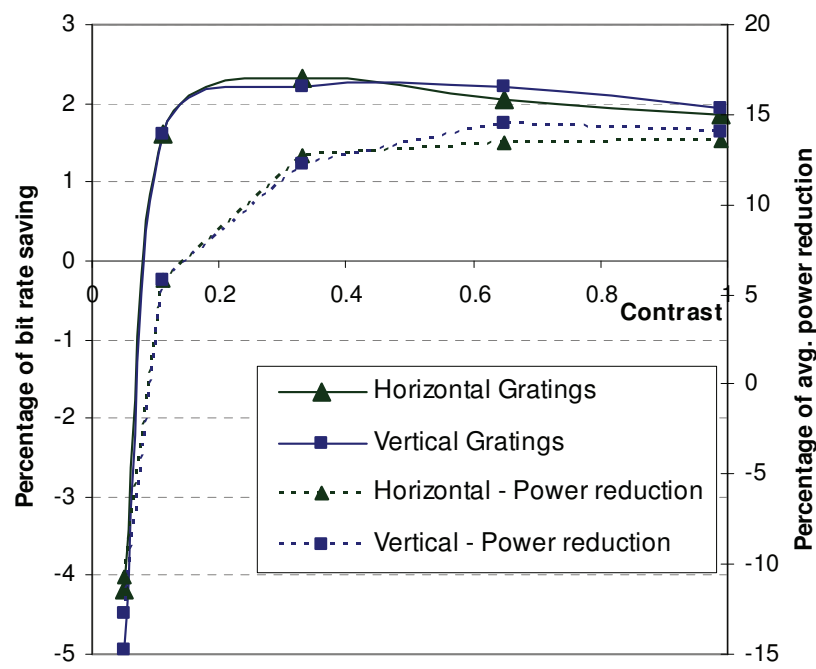

Figure 7. Achievable bit rate savings at different luminance contrasts

\section{B. Effect of ocular dominance on IBS tolerance}

This subsection briefly analyze the effect of ocular dominance towards IBS tolerance.

Among the participants, 6 subjects were left eye dominance and 10 subjects were right eye dominant. The variation of IBS Tolerance with spatial frequency is illustrated in Fig. 8. For clarity of presentation, standard deviation not shown in Fig. 8, however, it varied between 1.3 units to 0.6 units at different data points without any significant pattern. According to the results, the left eye dominant subjects tend to be less tolerant to asymmetric blur than the right eye dominant subjects.

C. Comparison of asymmetric blurring and asymmetric coding of stereoscopic images

Image or video compression techniques yields in several types of artifacts, such as blurring, blocking and ringing artifacts [11]. Due to the quantization of high frequency components, there is blurring of the image. Furthermore, due

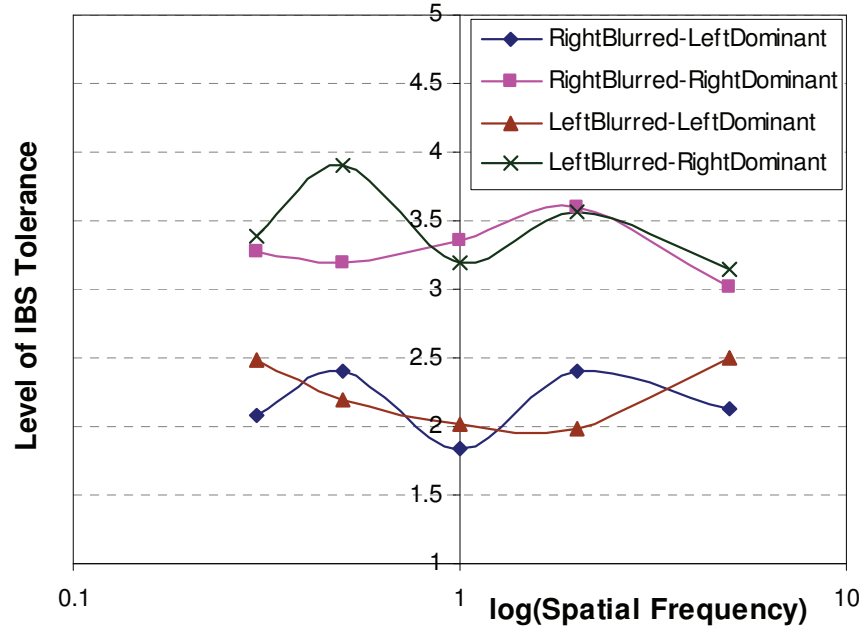

Figure 8. Effect of ocular dominance on IBS tolerance

Table II

BIT RATE REDUCTIONS ACHIEVED BY THE TWO ASYMMETRIC PROCESSING SCHEMES

\begin{tabular}{|l|l|c|c|c|c|}
\hline \multirow{2}{*}{ Image } & \multirow{2}{*}{$\begin{array}{l}\text { Left View } \\
\text { Bit rate } \\
(\mathrm{kbps}) \text { for }\end{array}$} & \multicolumn{4}{|c|}{ Right View } \\
\cline { 3 - 6 } & $40 \mathrm{~dB}$ & \multicolumn{2}{|c|}{$\begin{array}{c}\text { Quantization } \\
\text { (kbps) }\end{array}$} & & \multicolumn{2}{c|}{ Gaussian Blurring } \\
\hline Badminton & 20321 & 5159 & 74.61 & $\begin{array}{c}\text { Bit rate } \\
(\mathrm{kbps})\end{array}$ & $\Delta \mathrm{BR} \%$ \\
\hline Beergarden & 28768 & 7017 & 75.61 & 2288 & 80.5 \\
\hline Cafe & 3472 & 1692 & 51.26 & 1022 & 70.6 \\
\hline GT Fly & 5471 & 4420 & 19.21 & 561 & 89.7 \\
\hline
\end{tabular}

to the block based architecture of standardized video codecs, such as H.264/AVC, there are blocking artifacts, which appear as edges, especially in areas of low spatial frequencies.

The results of the subjective experiment described in section III further highlights the difference between blurring and quantization. To illustrate the difference of the two asymmetric processing schemes we summarize the bit rate reductions that is achievable in Table II. Accordingly it is clear that achieving stereoscopic asymmetry in terms of blurring is more effective in terms of subjective quality.

The level of blur at the point of just noticed difference, objectively measured in terms of Average Edge Width (AEW) [12], in the two cases is illustrated in Fig.9. It is clear that amount of blur present, when asymmetry is identified in asymmetric compression is very much lower than the case of asymmetric blurring. The high standard deviation for case of Gaussian blurring indicate that just noticeable point with asymmetric blurring varied significantly among individuals. However, in the case of quantization, most of the viewers agreed on the point of just noticeable difference, which was mainly identified by the visibility of blocking artifacts.

Most of the recently reported subjective assessments [5][6][7] were carried out by encoding the video at various quantization parameters and varying the level of quantization until subjects perceive a difference. However, as described above, the video coding artifacts consists of two parts, blur- 


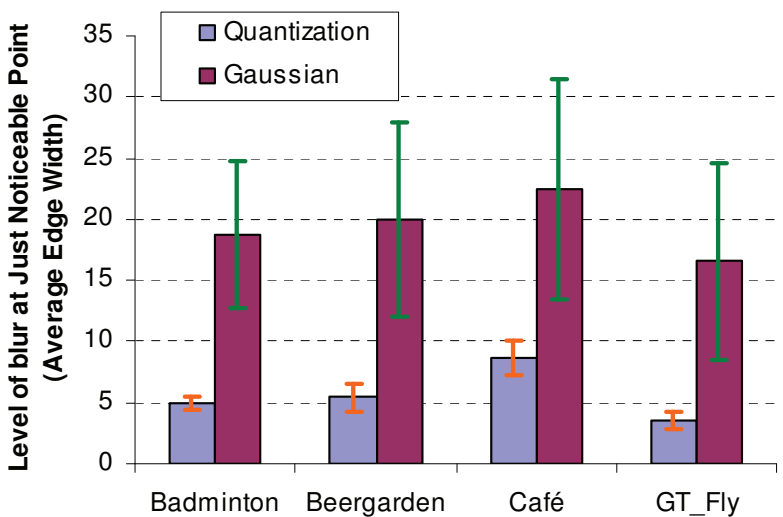

Figure 9. Comparison of objective measurement of blur at just noticeable point in quantization and Gaussian blurring

ring and blocking artifacts. The psycho-physical experiment reported in this paper suggests that humans can tolerate a significant amount of asymmetric blur before perceiving a difference. This level of tolerance is much higher than the level of blur that is present in just noticeable asymmetrically encoded video sequences [7]. Thus, the low tolerance of asymmetric encoding as compared to asymmetric blurring is mainly attributed to the blocking artifacts.

The human visual system (HVS) can successfully perceive high spatial frequencies of a stereoscopic image pair, masking any blur. Blocking artifacts introduce high spatial frequencies in to the stereoscopic pairs, where as Gaussian blurring reduces high spatial frequencies. Therefore, the effect of blocking artifacts are perceived in stereoscopic viewing, but the effect of blurring is not perceived. If there are blocking artifacts in a certain area of one view of a stereo pair, and if they are not present in the corresponding area of the other view, this gives rise to binocular rivalry as described in the introduction. It is reasonable to assume that subjects identify the level of asymmetric encoding by way of blocking artifacts, rather than by the asymmetric level of blur.

\section{Conclusions}

This paper presented results of a series of psycho-physical experiments performed to identify the limits of binocular suppression in stereoscopic images. Specifically, psycho-physical limits of interocular blur suppression is measured at various spatial frequencies, luminance contrasts and orientations. Results indicated that irrespective of the spatial frequency or the luminance contrast, the subjects are able to tolerate blur in horizontal gratings than of vertical gratings. In general left eye dominant subjects tend to be more sensitive to asymmetric blurring than right eye dominant viewers.

A further subjective experiment is carried out to illustrate the difference between two processing mechanisms, Gaussian blurring and quantization, that are used in asymmetric stereoscopic video compression. The subjective results indicate that viewers could relatively easily identify stereoscopic asymme- try achieved by quantization, than asymmetry achieved by Gaussian blurring. The main reason for this difference is attributed to the blocking artifacts that are visible in compressed images, which appear as high spatial frequency noise. The results of this paper reiterate the fact that HVS could perceive high spatial frequencies of a stereoscopic image pair, and thus clearly perceiving the effect of blocking artifacts, whereas the effect of Gaussian blurring is masked.

In the future, we will consider psycho-physical experiments that measure the just noticeable level of binocular rivalry, which could be used to objectively predict the subjective quality of asymmetric stereoscopic video. Furthermore, considering the bit rate savings that is achievable by asymmetric blurring, it is possible to develop a spatial frequency adaptive filter to pre-process the stereoscopic content to reduce the bit rate required for $3 \mathrm{D}$ video delivery.

\section{ACKNOWLEDGMENTS}

The work reported in this paper is funded by the 'ROMEO' Project funded by the European Union's $7^{\text {th }}$ Framework Program.

\section{REFERENCES}

[1] B. Julesz, "Cyclopean perception and neurophysiology," Investigative Ophthalmology \& Visual Science, vol. 11, no. 6, p. 540, 1972.

[2] M. G. Perkins, "Data compression of stereopairs," IEEE Transactions on Communications, vol. 40, no. 4, pp. 684-696, Apr. 1992.

[3] W. D. Reynolds and R. V. Kenyon, "The wavelet transform and the suppression theory of binocular vision for stereo image compression," in , International Conference on Image Processing, 1996. Proceedings, vol. 1. IEEE, Sep. 1996, pp. 557-560 vol.2.

[4] L. Lei and C. M. Schor, "The spatial properties of binocular suppression zone," Vision research, vol. 34, no. 7, pp. 937-947, 1994.

[5] H. Kalva, L. Christodoulou, L. M. Mayron, O. Marques, and B. Furht, "Design and evaluation of a 3D video system based on h.264 view coding," in Proceedings of the 2006 international workshop on Network and operating systems support for digital audio and video, ser. NOSSDAV '06. Newport, Rhode Island: ACM, 2006, pp. 12:1-12:6, ACM ID: 1378207.

[6] P. Aflaki, M. M. Hannuksela, J. Hakkinen, P. Lindroos, and M. Gabbouj, "Subjective study on compressed asymmetric stereoscopic video," in Image Processing (ICIP), 2010 17th IEEE International Conference on, pp. 4021-4024

[7] G. Saygili, C. G. Gurler, and A. M. Tekalp, "Evaluation of asymmetric stereo video coding and rate scaling for adaptive 3D video streaming," IEEE Transactions on Broadcasting, vol. 57, no. 2, pp. 593-601, Jun. 2011.

[8] G. Saygili, C. G. GuÎ́Lrler, and A. M. Tekalp, "Quality assessment of asymmetric stereo video coding," in 2010 17th IEEE International Conference on Image Processing (ICIP). IEEE, Sep. 2010, pp. 40094012.

[9] M. J. Collins and A. Goode, "Interocular blur suppression and monovision," Acta Ophthalmologica, vol. 72, no. 3, pp. 376-380, April 1995. [Online]. Available: http://eprints.qut.edu.au/854/

[10] J. Proakis, Digital Communications, 4th ed. 4th Edition, McGraw Hill, 2000.

[11] A. Leontaris and A. R. Reibman, "Comparison of blocking and blurring metrics for video compression," in Acoustics, Speech and Signal Processing, 2005. ICASSP 2005. IEEE International Conference on, 2005, pp. $585-588$.

[12] S. W. Pina Marziliano, Frédéric Dufaux and T. Ebrahimi, "A noreference perceptual blur metric," in Image Processing, 2002. ICIP '02. 2002 International Conference on, vol. 3, 2002, pp. 57-60 Vol. 3. 